\title{
¿También somos variables ante la Evidencia?
}

\author{
Fernando Moreno García ${ }^{a}, M^{a}$ Amparo Sánchez Ramiro ${ }^{a}$, Gemma Alejandre Lázaroa, \\ Olga Rubio Pu lido ${ }^{\mathrm{b}}$, Laura Fernández Agüero ${ }^{\mathrm{b}}$, Alejandro Villarín Castroc, \\ $M^{a}$ Gracia Viseu Pinheiro Lopes do Rego ${ }^{a}$
}

\begin{abstract}
${ }^{a}$ Médico de Familia. Gerencia de Atención Primaria de Toledo.
\end{abstract}

${ }^{\text {b }}$ Farmacéutica. Gerencia de Atención Primaria de Toledo.

c Médico de Familia. Unidad Docente de Medicina de Familia y Comunitaria de Toledo.

Correspondencia:

Fernando Moreno García,

$\mathrm{C} /$ Barcelona $\mathrm{n}^{\circ} 2$,

45005 - Toledo, España.

Telf.: 925259925 ,

fax: 925215450 , e-mail:

fmgarcia@sescam.jccm.es.

Recibido el 12 de octubre de 2009.

Aceptado para su publicación el 10 de enero de 2010.

\section{RESUMEN}

Objetivo. Describir la variabilidad terapéutica entre médicos de Atención Primaria (AP) ante casos clínicos con evidencia respecto a su tratamiento.

Diseño. Descriptivo y transversal.

Emplazamiento. Área de Salud de Toledo.

Participantes. Médicos de familia con contrato estable.

Mediciones principales. Encuesta anónima y autocumplimentada con 6 casos clínicos frecuentes: 1) Neumonía típica adquirida en la Comunidad, varón joven sin complicaciones (tratamiento según evidencia: amoxicilina), 2) Herpes zoster oftálmico (antiviral + paracetamol/codeína), 3) Artrosis en posmenopáusica sin osteoporosis (paracetamol), 4) Cistitis, mujer joven sin complicaciones (amoxicilina-clavulánico, norfloxacino y fosfomicina), 5) gastroprotección en paciente con corticoides (no), 6) Prevención trombosis venosa profunda, varón joven sin factores de riesgo con esguince de tobillo (no heparina de bajo peso molecular).

Se recogieron datos del médico (edad, sexo, MIR, años de experiencia) y entorno laboral (contrato, presión asistencial, cupo, distancia al hospital).

Resultados. $\mathrm{N}=146$ (tasa de respuesta $51 \%$ ). Caso 1: tratamiento según evidencia $6,8 \%$ (IC 95\%: 4,7-8,9); Caso 2: 31,5\% (IC 95\%: 27,7-35,3); Caso 3: 80,8\% (IC 95\%: 77,5-84,1); Caso 4: $90,5 \%$ (IC 95\%: 88,1-92,9); Caso 5: 15,9\% (IC 95\%: 12,9-18,9); Caso 6: $72,5 \%$ (IC $95 \%$ : $68,7-76,2)$. Las variables sexo, contrato, cupo, distancia al hospital y presión asistencial no se relacionaron con la respuesta. En el caso 2 se encontraron diferencias estadísticamente significativas respecto a edad $(p<0,0001)$ y experiencia $(p<0,01)$. En los casos 1 y 5 sólo para formación MIR ( $p<0,05$ y $p<0,005$ respectivamente).

Conclusiones. Constatamos variabilidad en la prescripción incluso ante situaciones para las que existe evidencia científica. Muchas de las actitudes terapéuticas no coinciden con la mayoría de las recomendaciones. El mejor conocimiento de la práctica clínica es fundamental para aumentar la calidad asistencial, al detectar áreas de mejora para las que priorizar actuaciones.

Palabras clave. Prescripción de Medicamentos, Atención Primaria de Salud.

\section{ABSTRACT}

Do we also vary when faced with evidence based treatment?

Objective. To determine treatment variability among Primary Care (PC) doctors using clinical cases with evidence based treatment.

Design. Descriptive, cross-sectional study.

Setting. Toledo Health Area

Participants. General practitioners with a stable contract.

Main measurements. Self-complete, anonymous questionnaire on six common clinical cases: 1) Community acquired pneumonia, young maln with no complications (evidence based treatment: amoxicillin), 2) Herpes zoster ophthalmicus (antiviral + paracetamol/codeine), 3) Osteoarthritis in post-menopause without osteoporosis (paracetamol), 4) Cystitis, young woman with no complications (amoxicillin-clavulanic acid, norfloxacin and phosphomycin), 5) gastroprotection in patient taking corticoids (no), 6) Prevention of deep vein thrombosis, young man with no risk factors and a sprained ankle (not low molecular weight heparin).

The doctors' characteristics were collected (age, sex, medical residency training (MIR), years of experience) work conditions (contract, attendance pressure, quota, distance from hospital). Results. $\mathrm{N}=146$ (response rate $51 \%)$. Case 1: evidence based treatment $6.8 \%(95 \% \mathrm{Cl}: 4.7-$ 8.9); Case 2: $31.5 \%$ (95\%Cl: 27.7-35.3); Case 3: 80.8\% (95\%Cl: $77.5-84.1$ ); Case 4: $90.5 \%$ (95\%Cl: 88.1-92.9); Case 5: 15.9\% (95\% Cl: 12.9-18.9); Case 6: 72.5\% (95\% Cl: 68.7-76.2). The variables of sex, contract, distance from hospital and attendance pressure were not related to response. In case 2 statistically significant differences were found with respect to age, $(p<0.0001)$ and experience $(p<0.01)$ and in cases 1 and 5 only with respect to MIR $(p<0.05$ and $p<0.005$ respectively).

Conclusions. We found prescription variability even in clinical situations for which there was scientific evidence. Many of the treatment habits did not coincide with most of the recommendations. Better knowledge of clinical practice is essential to improve healthcare quality. Areas for improvement need to be detected in order to prioritize actions.

Key words. Drug Prescriptions, Primary Health Care. 
Este estudio fue presentado como comunicación oral en el XXV Congreso de la Sociedad Española de Calidad Asistencial, celebrado en Barcelona durante los días 16 a 19 de octubre de 2007.

"Experiencia es el nombre que damos a nuestros errores"

(Óscar Wilde)

\section{INTRODUCCIÓN}

El concepto de variabilidad en la práctica clínica ya fue utilizado por Alison Glover en $1938^{1}$ como un factor que abría serios interrogantes sobre la naturaleza última de la toma de decisiones en medicina y su adecuación. El aforismo hipocrático "no hay enfermedades sino enfermos" es interiorizado por los profesionales de la medicina desde el comienzo de su formación, reconociéndose implícitamente que la toma de decisiones ante un paciente está sujeta a incertidumbre. Por lo tanto, se asume que la variabilidad forma parte inseparable de la práctica clínica. Los numerosos estudios realizados con posterioridad han demostrado que la variabilidad sigue estando presente en la praxis médica, casi como una constante, siete décadas después.

Con la aparición de la "Medicina Basada en la Evidencia" en el último tercio del siglo XX, la medicina clínica incorpora el mejor nivel de evidencia científica a la toma de decisiones, pasándose del experiencialismo (la medicina clínica como arte) al experimentalismo (la medicina clínica como ciencia) ${ }^{2}$. Si la medicina clínica pasa a tener su base en el método científico, no estaría justificada la variabilidad cuando existe evidencia. A pesar de ello, en las consultas, en las plantas de hospital y en los servicios de urgencia observamos que, incluso ante el mejor nivel de conocimiento científico, parece seguir existiendo cierto grado de variabilidad a la hora de aplicarlo a los problemas de salud.

Comprobar la existencia de esta variabilidad y en qué medida se produce es fundamental para tratar de racionalizar los múltiples factores que intervienen en la adopción de decisiones diferentes sobre personas que padecen la misma enfermedad. Esto es importante porque lo que ha dado en llamarse "variabilidad no deseable" puede llevar detrás una atención sanitaria de mala calidad ${ }^{3}$, y su identificación nos permitiría detectar áreas de mejora en la atención a los pacientes.

La importancia económica y sociosanitaria que la prescripción de fármacos tiene para los servicios de salud, el hecho de que buena parte de la evidencia científica se genera en torno al tratamiento de las enfermedades y la importancia creciente de la seguridad clínica hacen que la prescripción sea un elemento clave en los estudios de variabilidad ${ }^{4}$.
El objetivo del presente estudio fue describir la variabilidad en la prescripción realizada por médicos de Atención Primaria (AP) ante casos clínicos para los que existe evidencia científica sobre su tratamiento.

\section{MATERIAL Y MÉTODO}

Se trata de un estudio observacional, descriptivo y transversal, basado en una encuesta anónima y autocumplimentada, elaborada "ad hoc", realizado entre los meses de marzo y abril de 2007. La población de estudio fue el conjunto de médicos de familia de AP del Área de Salud de Toledo con contrato de larga duración. El cuestionario fue remitido de forma personalizada por correo interno a cada uno de los facultativos del área, junto a un sobre de respuesta ya cumplimentado para su devolución, también por correo interno. Se realizó un doble envío para aumentar la tasa de respuestas.

La encuesta fue confeccionada con 6 casos clínicos más una serie de preguntas sobre factores que a priori podrían estar relacionadas con la variabilidad en las respuestas. En cada uno de los casos se ofrecían varias opciones terapéuticas relacionadas con el tema en cuestión. Los tratamientos considerados correctos se basaron en la evidencia científica disponible en ese momento. Se llevó a cabo un pilotaje previo con una muestra de 15 facultativos.

Los casos clínicos sobre los que se preguntó versaban sobre los siguientes temas:

Caso 1: Neumonía típica adquirida en la comunidad en varón joven sin complicaciones (tratamiento correcto: amoxicilina ${ }^{5}$ ).

Caso 2: Herpes zóster en rama del trigémino (tratamiento correcto: antiviral + codeína-paraceta$\left.\mathrm{mol}^{6-7}\right)$.

Caso 3: Artrosis en postmenopáusica sin osteoporosis (tratamiento correcto: paracetamol + medidas higiénicas ${ }^{5}$ ).

Caso 4: Cistitis no complicada en mujer joven (tratamiento correcto: fosfomicina trometamol, amoxicilina-clavulánico, norfloxacino $0^{5,8}$ ).

Caso 5: Gastroprotección en paciente de menos de 65 años en tratamiento con corticoides (tratamiento correcto: no precisa gastroprotección ${ }^{9}$ ).

Caso 6: Prevención de trombosis venosa profunda (TVP) en varón joven sin factores de riesgo con esguince de tobillo (tratamiento correcto: no precisa heparina de bajo peso molecular ${ }^{10}$ ). 
Se consideraron como variables principales las respuestas a los distintos casos clínicos planteados. Las variables independientes recogidas en el mismo cuestionario fueron la edad y sexo del médico, formación MIR y años de experiencia profesional, el tipo de contrato de vinculación con el Servicio de Salud, la presión asistencial media de la consulta o punto de atención continuada, el tamaño del cupo, si era pertinente, y la distancia al hospital de referencia.

Todos los datos fueron introducidos en una base de datos creada al efecto y analizados mediante el programa estadístico SPSS 12.0 para Windows. Para el análisis se utilizaron las medidas de tendencia central y dispersión en el caso de variables cuantitativas y la distribución de frecuencias para las cualitativas. Los intervalos de confianza se calcularon al $95 \%$. Los test de contraste de hipótesis usados fuero la t de Student para las variables cuantitativas y la chi-cuadrado para las cualitativas.

\section{RESULTADOS}

Se obtuvieron 146 respuestas, lo que representa una tasa de respuesta del $51 \%$. En cuanto a las características sociodemográficas, la edad media de los participantes era de 45,41 años (DE: 8,22), pertenecía al sexo masculino el $56,3 \%$, el $41,1 \%$ tenía formación MIR y el número medio de años de experiencia profesional era de 19,03 años (DE: $8,67)$. Respecto al tipo de contrato, eran titulares el $53,7 \%$, sustitutos el $6,6 \%$, interinos el $22,8 \%$ y Profesionales Estatutarios de Atención Continuada (PEAC) el 16,9\%. La presión asistencial media reconocida por los facultativos fue de 53,05 pacientes/día (DE: 17,3). El tamaño medio del cupo de las consultas fue de 1.531,11 Tarjetas Sanitarias Individuales (DE: 477,5). La distancia media al hospital fue de $32,74 \mathrm{~km}$ (DE: 23,11).

Los porcentajes de respuestas correctas, según la evidencia disponible, se indican a continuación:

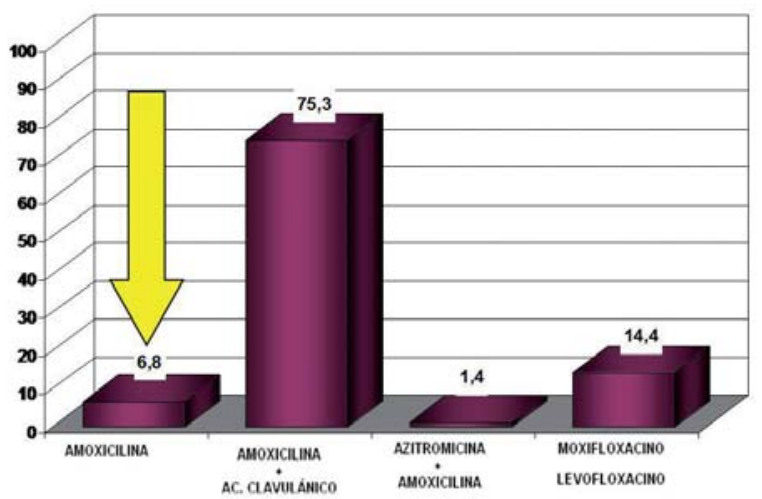

Figura 1. Respuestas obtenidas en el caso 1: neumonía adquirida en la comunidad en varón joven sin complicaciones. La flecha señala la respuesta correcta.
$6,8 \%$ en el caso 1 (IC 95\%: 4,7-8,9), 31,5\% en el caso 2 (IC 95\%: $27.7-35,3$ ), $80,8 \%$ en el caso 3 (IC 95\%: 77,5-84,1), 90,5\% en el caso 4 (IC 95\%: $88,1-92,9), 15,9 \%$ en el caso 5 (IC 95\%: 12,9-18,9) y $72,5 \%$ en el caso 6 (IC 95\%: 68,7-76,2). En las figuras 1 a 6 aparecen reflejados todos los porcentajes de respuestas a las diferentes opciones de cada caso clínico.

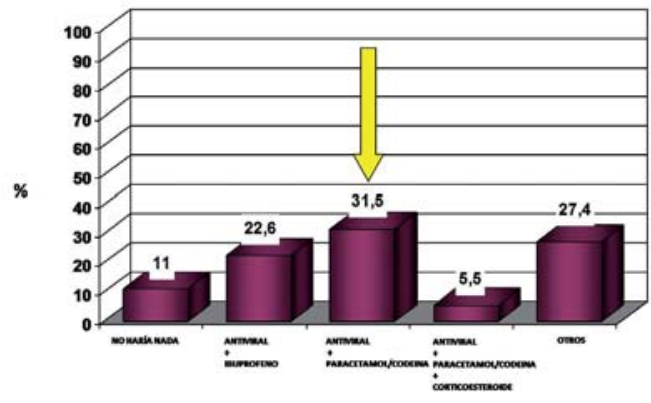

Figura 2. Respuestas obtenidas en el caso 2: herpes zoster en territorio del trigémino. La flecha señala la respuesta correcta.

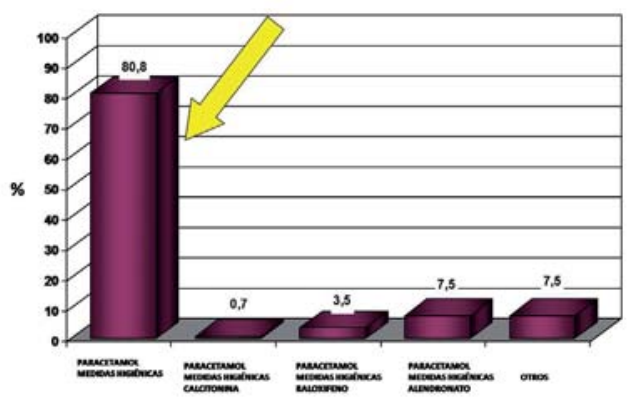

Figura 3. Respuestas obtenidas en el caso 3: artrosis en postmenopáusica sin osteoporosis. La flecha señala la respuesta correcta.

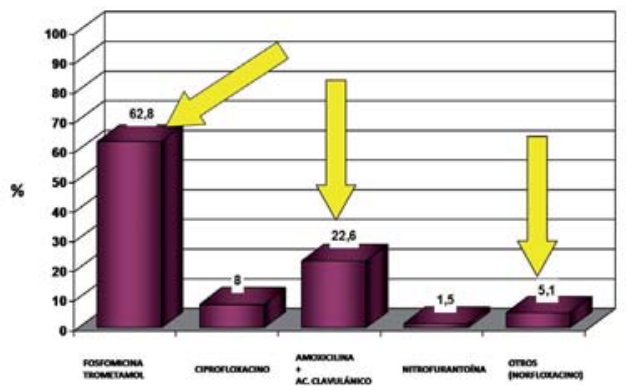

Figura 4. Respuestas obtenidas en el caso 4: cistitis aguda no complicada en mujer joven. La flecha señala la respuesta correcta.

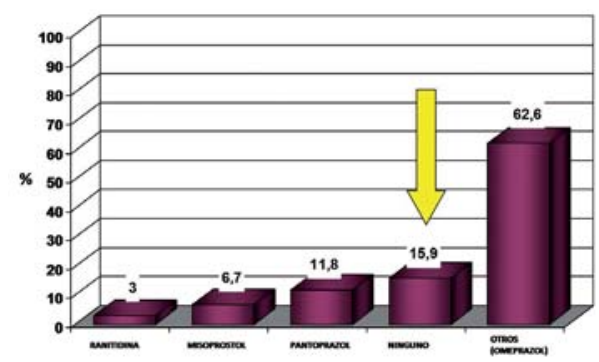

Figura 5. Respuestas obtenidas en el caso 5: gastroprotección en un paciente menor de 65 años en tratamiento con corticosteroides. La flecha señala la respuesta correcta. 


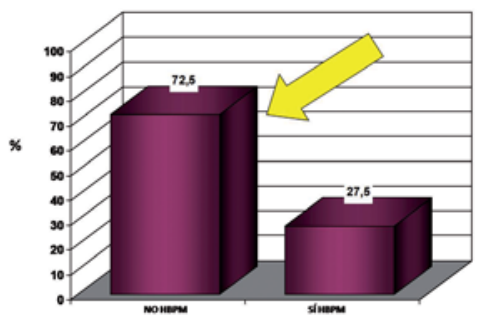

Figura 6. Respuestas obtenidas en el caso 6: prevención de TVP en varón joven sin factores de riesgo con esguince de tobillo. La flecha señala la respuesta correcta.

Considerando la totalidad de las respuestas ( $\mathrm{n}=$ 876), sólo el 49,7\% (IC 95\%: 48-52,4) era acorde a la evidencia científica actual. De forma global, el hecho de responder conforme a la evidencia no se relacionaba de forma significativa con las variables sexo, contrato, cupo, distancia al hospital y presión asistencial. No obstante, en el caso 2 (herpes zoster) sí se encontraron diferencias estadísticamente significativas respecto a la edad $(t=3,773 p<$ $0,0001)$ y a la experiencia $(t=2,852 p<0,01)$ a favor de los mayores de 40 años. En los casos de neumonía y gastroprotección se apreciaron diferencias respecto a la formación MIR ( $t=12,848 p$ $<0,05$ y $t=16,409 p<0,005$, respectivamente) a favor de los especialistas vía MIR.

\section{DISCUSIÓN}

La tasa de respuesta obtenida (51\%) nos parece más que aceptable, considerando que se trataba de un cuestionario autocumplimentado de remisión por correo. En cuanto a la representatividad de la muestra, las características sociodemográficas de la misma se corresponden adecuadamente con las de nuestra población.

En la revisión bibliográfica realizada sólo hemos encontrado trabajos sobre variabilidad en la prescripción, dirigidos fundamentalmente a dos aspectos: de forma genérica sobre un grupo terapéutico (antibióticos, psicofármacos, etc.) o sobre situaciones clínicas de incertidumbre. Por el contrario, nuestro estudio explora la variabilidad ante situaciones para las que sí existe evidencia, es decir, aquella variabilidad de la práctica clínica que podemos considerar como inaceptable.

En los escenarios planteados hemos encontrado una gran variación en el porcentaje de respuestas correctas, según el tema planteado en cada caso clínico. Así, donde más decisiones erróneas aparecen es en los supuestos con procesos infecciosos, a excepción del caso de la cistitis. Otros estudios ya han constatado cómo la prescripción de los antibióticos es uno de los procesos clínicos sujetos a mayor variabilidad, especialmente en nuestro país ${ }^{11}$. Esto se podría explicar porque, pese a existir una evidencia fundamentada en estudios clínicos, se han extendido diversidad de protocolos y supuestas guías de práctica clínica (documentos que no siguen la metodología de una guía pero que se propagan como tales) que no se ajustan a dichos estudios o a los niveles de evidencia derivados de ellos. Además de la gran dificultad que supone la implantación, en la práctica clínica diaria, de protocolos y guías clínicas.

Llaman la atención los resultados de los casos clínicos de gastroprotección y de prevención de la trombosis venosa profunda, donde existen evidencias ampliamente consensuadas. Entre las distintas causas reconocidas de variabilidad nos encontramos con la falta de difusión o conocimiento de la evidencia científica entre los profesionales. En este sentido, el mantenerse actualizado, aunque debe ser una obligación moral ó ética para los facultativos, no siempre es tan fácil como tendría que ser por múltiples razones: formación fuera del horario laboral, falta de sustitutos, exceso de horas de trabajo $^{12}$, etc. Los servicios de salud, hasta el momento, han sido incapaces de solventar estas dificultades, a pesar de tener la responsabilidad de ofrecer una formación continuada acorde a las necesidades.

En la misma línea consideramos importante la influencia de la industria farmacéutica, tanto durante la visita médica como en las actividades de formación continuada que organizan (generalmente más atractivas que las institucionales), y que, en muchos casos, constituyen la principal fuente de información de los profesionales. La extensión del uso de Internet y el correo electrónico puede ser herramientas imprescindibles que vengan a solucionar este problema. Así, la implantación generalizada de cursos on-line, las bibliotecas virtuales o los sistemas de alerta bibliográfica, entre otros, contribuirían de forma indiscutible a la difusión de las evidencias.

A pesar de las consideraciones anteriores, no hay que olvidar que otra de las causas más ampliamente defendidas de variabilidad es el denominado "estilo de práctica"4,13,14. Entendemos como "estilo de práctica" las diferentes opiniones de los facultativos sobre las ventajas e inconvenientes de las distintas opciones terapéuticas o diagnósticas para una patología dada ${ }^{14}$. Así, ante una determinada circunstancia clínica, no todos los médicos actuarían igual, y estas actitudes se mantendrían de forma sistemática. El apego de muchos profesionales a su "propio estilo" puede condicionar sus actuaciones por encima de protocolos y guías clínicas. Pero no hay que olvidar que ese "toque personal", tan importante en la interacción humana del médico de familia con el paciente, no debe anteponerse a la evidencia científica, ni encubrir una práctica clínica mediocre ${ }^{12}$. Las variables estudiadas dependientes del médi- 
co no parecen influir en la adecuación o no de las respuestas a la evidencia. Este hecho se contrapone a los resultados de los estudios sobre variabilidad en los casos de incertidumbre, donde las características individuales del médico si influyen en su actitud terapéutica ${ }^{13}$. Sin embargo, tanto en las investigaciones en situaciones de incertidumbre como en la nuestra, con evidencia disponible, las características del cupo no parecen mediar en los resultados ${ }^{13,15}$.

En resumen, casi la mitad de las actitudes terapéuticas no coinciden con las recomendaciones basadas en la evidencia. Esto nos debe plantear dudas sobre la calidad de la atención, tanto en términos de equidad (infrautilización en determinadas áreas de la salud) como de eficiencia (sobreutilización inadecuada en otras) ${ }^{14}$. La monitorización del trabajo clínico, con indicadores de adecuación a la evidencia (protocolos, guías o vías clínicas de calidad) que se determinen, tendría un papel trascendental en la disminución de la variabilidad clínica y en la mejora de la calidad asistencial.

A pesar de todo, la ciencia médica se desarrolla en el seno de una relación humana, donde el sujeto principal (el paciente) tiene sus propios significados y su contexto ${ }^{16}$. Y son estos, no otras causas, los únicos que pueden justificar la variabilidad cuando existe evidencia científica.

\section{BIBLIOGRAFÍA}

1. Glover JA. The incidence of tonsillectomy in school children. Proc R Soc Med. 1938; 31:1219-36.

2. Soriguer $F$. El fracaso de la investigación clínica en España. Med Clin. 2009; 132 (6): 219-21.

3. Delgado R, Carrasco G. Variabilidad no deseable en la práctica clínica. Rev Calidad Asistencial. 2000; 15:639-40.

4. López de Castro F, Montero MJ, Valles N, Fernández O, Ale- jandre G, Chacón J. Variabilidad en la prescripción farmacéutica de Atención Primaria de Castilla La Mancha durante 2003. Rev Esp Salud Pública. 2005; 79:551-8.

5. Guía farmacoterapéutica en Atención Primaria basada en la evidencia. $2^{\mathrm{a}}$ ed. SEMFYC; 2004.

6. Hervás Angulo A, Forcén Alonso T. Herpes zoster y neuralgia postherpética. Guía clínica de Fisterra. 2006; 6(29). [Consultado 3-2-2007]: Disponible en: http://www.fisterra.com/ guias2/Herpes.asp.

7. Lancaster T, Silagy C, Gray S. Primary care management of acute herpes zoster: systematic review of evidence from randomized controlled trials. Br J Gen Pract. 1995; 45:39-45.

8. González Gero MY, Muñoz Gavilán A. Uso adecuado de fluoroquinolonas en Atención Primaria. B Farmacoterapéutico de Castilla la Mancha. 2004; 5(1):1-8.

9. Lanas A, Martín-Mola E, Ponce J, Navarro F, Piqué JM, Blanco FJ. Estrategia clínica para la prevención de los efectos adversos sobre el tracto digestivo de los antiinflamatorios no esteroideos. Gastroenterol Hepatol. 2003; 26(8):487-504.

10. Alonso Ortiz del Río C, Medrano Ortega FJ, Romero Alonso A, Villar Conde E, Calderón Sandubete E, Marín León I y Cols. Guía PRETEMED 2003. Guía de profilaxis de enfermedad tromboembólica en patología médica. Córdoba: SADEMI; 2003.

11. Cars $O$, Mölstad S, Melander A. Variation in antibiotic use in the European Union. Lancet. 2001; 357(9271):1851-3.

12. Ojeda Pérez F. Variabilidad clínica. Una visión del profesional médico. Rev Calidad Asistencial. 2006; 21(2):63-5.

13. Moreno E, López F, Llamas MA, Mareque MA, Manzano M, Martín M. Actitud de los médicos de Atención Primaria ante situaciones de incertidumbre. Rev Clin Med Fam. 2007; 2(2):51-6.

14. Marion Buen J, Peiro S, Márquez Calderón S, Meneu de Guillerna R. Variaciones en la práctica médica: importancia, causas e implicaciones. Med Clin (Barc). 1998; 110:382-90.

15. Vega AT, Larrañaga M, Zurriaga O, Gil M, Urtiaga M, Calabuig J. Trastornos de ansiedad. Variabilidad y condicionantes de la actitud terapéutica de los médicos de atención primaria. Aten Primaria. 1999; 24:569-78.

16. Turabián Fernández JL, Pérez Franco B. La variabilidad es un indicador de buena gestión clínica en medicina de familia. Aten Primaria. 2006; 37(3):160-3. 